(C) 2006 International Press

Adv. Theor. Math. Phys. 10 (2006) 159-179

\title{
Inherited duality and quiver gauge theory
}

Nick Halmagyi, Christian Römelsberger, and Nicholas P. Warner

Department of Physics and Astronomy, University of Southern California, Los Angeles, CA 90089, USA

halmagyi, romelsberger, warner@usc.edu

\begin{abstract}
We study the duality group of $\widehat{A}_{n-1}$ quiver gauge theories, primarily using their M5-brane construction. For $\mathcal{N}=2$ supersymmetry, this duality group was first noted by Witten to be the mapping class group of a torus with $n$ punctures. We find that it is a certain quotient of this group that acts faithfully on gauge couplings. This quotient group contains the affine Weyl group of $\widehat{A}_{n-1}, \mathbb{Z}_{n}$ and $S L(2, \mathbb{Z})$. In fact there are $n$ non-commuting $S L(2, \mathbb{Z})$ subgroups, related to each other by conjugation using the $\mathbb{Z}_{n}$. When supersymmetry is broken to $\mathcal{N}=1$ by masses for the adjoint chiral superfields, a renormalization group (RG) flow ensues which is believed to terminate at a conformal field theory (CFT) in the infrared. We find the explicit action of this duality group for small values of the adjoint masses, paying special attention to when the sum of the masses is non-zero. In the $\mathcal{N}=1 \mathrm{CFT}$, Seiberg duality acts nontrivially on both gauge couplings and superpotential couplings and we interpret this duality as inherited from the $\mathcal{N}=2$ parent theory. We conjecture the action of $S$-duality in the CFT based on our results for small mass deformations. We also consider non-conformal deformations of these $\mathcal{N}=1$ theories. The cascading RG flows that ensue are a oneparameter generalization of those found by Klebanov and Strassler and by Cachazo et al. The universality exhibited by these flows is shown to be a simple consequence of paths generated by the action of the affine Weyl group.
\end{abstract}

e-print archive: http://lanl.arXiv.org/abs/hep-th/0406143 


\section{Introduction}

For nearly a decade, branes have provided crucial insights into supersymmetric gauge theories. Two arenas where branes have found particular success are Maldacena's gauge/gravity duality [1] and IIA suspended brane constructions [2-4]. The duality has proved to be a useful arena for analyzing renormalization group ( $\mathrm{RG}$ ) flows, in many cases exact supergravity solutions can be found [5-7]. In ten dimensions these flows are driven by non-trivial profiles for the $p$-form fluxes and/or geometric moduli. The IIA constructions on the other hand, have proved perhaps a little less useful, at least in terms of RG flows but in this paper we will find great utility for these setups in uncovering explicit actions of duality groups.

With the goal of studying duality symmetries within a certain class of RG flows, we study the $\mathcal{N}=2$ quiver gauge theory that is dual to IIB string theory on $A d S_{5} \times S^{5} / \mathbb{Z}_{n}[8,9]$. Five-dimensional, $\mathcal{N}=8$ gauged supergravity has proven very useful in the analysis of holographic flows in $\mathcal{N}=4$ Yang-Mills. It was argued in [11] that $\mathcal{N}=4$ gauged supergravity, with very specific couplings to $\mathcal{N}=4$ vector and tensor multiplets would provide a similar tool for $\mathcal{N}=2$ quiver gauge theories. The most general such supergravity theory was constructed in [10], and the precise version relevant to quiver gauge theories was found and analyzed in [11]. It is believed (but not proven) that this theory is a consistent truncation of the ten-dimensional theory, and so results derived in five dimensions should precisely correspond to ten-dimensional backgrounds. The results of [11] thereby made some intriguing predictions for IIB supergravity backgrounds. In particular, it was shown that, after gauging, there is an $S U(1, n)$ global symmetry group that remains unbroken. The $S U(1, n)$ acts on scalars holographically dual to the complex gauge couplings of each gauge group, implying that each gauge coupling can be set to an arbitrary value. (In fact the $S U(1, n)$ acts on more scalars than just those dual to gauge couplings. This will be described in more detail below.). In the untwisted IIB string theory on $A d S_{5} \times S^{5}$, this duality group is simply the $S U(1,1) \equiv S L(2, \mathbb{R})$ which is broken to $S L(2, \mathbb{Z})$ in string theory, and hence in the finite $N$ gauge theory. Thus one must expect, in general, that the $S U(1, n)$ will be broken to a discrete subgroup for finite-rank gauge groups. This led us to the present investigation of duality symmetries from the perspective of field theory.

There is another description of these $\mathcal{N}=2$ quiver gauge theories $[2,4]$ that is T-dual to the IIB description. This construction is the classic IIA suspended brane construction lifted to $M$-theory. In this construction, the D4-branes and the NS5-branes that they are suspended between, lift to $M$-theory as a single M5-brane wrapped on the Seiberg-Witten curve. 
For our purposes, the advantage of this $M$-theory picture over its IIB counterpart is that the duality symmetries can be easily and precisely described without the need to take a 't Hooft limit. In [4] Witten noted that the duality group that acts on complexified gauge couplings is the mapping class group of a torus with $n$ punctures, which following the notation used in [12] we denote $M(1, n)$.

The group $M(1, n)$ has been much studied by mathematicians and is known to be an extension of the torus braid group on $n$-strands by $S L(2, \mathbb{Z})$ [12]. We find that the representation of this group which acts on gauge couplings is not faithful but it fails to be faithful in a very simple way. If one supplements $M(1, n)$ with the condition that permutations are involutions instead of braiding, i.e., $s_{i}^{2}=1$ where $s_{i}$ exchanges the $i$-th and $i+1$-th punctures, then one does obtain a faithful representation. With these extra relations this new group has the following subgroups: the $\widehat{A}_{n-1}$ affine Weyl group, a $\mathbb{Z}_{n}$ which rotates the nodes of the quiver and of course $S L(2, \mathbb{Z})$. It has already been suggested that affine quiver theories have the affine Weyl group as a duality group [13], it is nice to find a direct connection to the $M$-theory picture. We find that the $S L(2, \mathbb{Z})$ has a preferred node and thus there are $n$ copies of $S L(2, \mathbb{Z})$ related by conjugation by elements of $\mathbb{Z}_{n}$. These three subgroups appear in an identical fashion in the context of $S U(n)$ WZW models, this is a tantalizing observation but we are unable to provide a deeper connection.

These field theories admit a family of relevant deformations by mass terms for adjoint chiral superfields, which according to the methods of Leigh and Strassler [14] should flow to a non-trivial CFT in the IR [11, 15]. The main purpose of this paper is to study the duality group of these flows. For the $\mathbb{Z}_{n}$ orbifold there are thus $n$ independent mass parameters. One of these mass parameters is the sum of the masses on the nodes of the quiver and, being invariant under permutations, it comes from the untwisted sector of the gauge theory. Such a deformation of the gauge theory is therefore is dual to the $\mathbb{Z}_{n}$ orbifold of the Pilch-Warner flow [6]. The other mass parameters are dual to complexified Kähler moduli of the blow-ups of the orbifold singularities [11]. For $n=2$, the IR fixed point of the resulting flow is dual to $A d S_{5} \times T_{1,1}$ [16], and the twisted-sector flows for the general orbifolds were described in [17]. One may also consider a relevant deformation by a combination of masses in both the twisted and untwisted sectors, and these too should flow to a CFT in the IR [11] however the ten-dimensional supergravity dual for these IR fixed points, let alone the entire flows has yet to be constructed.

On the other hand, while the potentials for five-dimensional gauged supergravity theories tend to be unwieldy, the analysis of special sub-sectors can 
prove relatively simple. For flows described above it was easy to show [11] that the $S U(1, n)$ invariance of the theory also acts on the scalars dual to adjoint masses. In particular, that the compact, $S U(n)$ subgroup acts on them in the fundamental representation. The holographic dual of the flow involving only the untwisted sector mass is explicitly known, both in five dimensions [5], and in ten-dimensions [6]. One can thus use the $S U(n)$ symmetry to map the known flow of [5] to any flow involving any combination of twisted and untwisted sector masses. This symmetry is manifest in five dimensions, but is far from obvious in ten dimensions: the untwisted sector mass is dual to a topologically trivial $B$-field flux, while the twisted sector masses involve Kähler moduli of blow-ups. Such an unusual duality rotation was one of the more intriguing geometric predictions of [11].

In the IIA picture, masses for the adjoint fields are introduced by rotating the NS5-branes [18] and explicit formulas in terms of the ten-dimensional geometry have been obtained when the masses are small. Using this, we are able to describe the duality symmetries of these mass deformations and again we find the affine Weyl group and $\mathbb{Z}_{n}$, however, only the $S$-transformation from $S L(2, \mathbb{Z})$ acts non-trivially. Each mass behaves under a Weyl reflection as a Dynkin label and the sum of the masses, the "global mass", is the level and is thus invariant.

As mentioned above, the RG flows that ensue from these mass deformations should all go to non-trivial, conformal fixed points. Indeed, the holographic solution [11] suggests that the fixed line of [14] becomes a family of such lines parameterized by $\mathbb{C P}^{n-1}=S U(n) /(S U(n-1) \times U(1))$. By integrating out the massive fields we get a quartic superpotential and a new set of superpotential couplings, which are proportional to the inverse of the masses. In the suspended brane construction, Seiberg duality corresponds to interchanging NS5-branes [19]. In [15] it was shown that Seiberg duality acts non-trivially on the superpotential couplings and a 1-parameter generalization of the Klebanov-Strassler cascading RG flow to include non-trivial profiles for the 3 -form flux was conjectured. These new flows were shown to exhibit universality in the sense that after a large number of Seiberg dualities, the sum of the inverse of the superpotential couplings approaches zero, which is the condition for vanishing 3 -form flux. In this paper we point out that the inverses of superpotential couplings transform under Seiberg duality as Dynkin labels under an affine Weyl reflection. The universality is then a simple statement about paths generated by the affine Weyl group. We interpret this duality as "inherited" from the parent $\mathcal{N}=2$ theory.

Inherited dualities are an interesting phenomenon [20]. The concept first appeared as an attempt to prove that in special cases, namely when $N_{f}=2 N_{c}$, Seiberg duality is inherited from $\mathcal{N}=2 S$-duality [14, 23]. Since 
then, $S$-duality of the $N=1^{*}$ flow has been intensively studied [22, 23]. The authors of [23] study $\mathcal{N}=2$ quiver gauge theories with non-zero masses for the hypermultiplets as well for the adjoint chiral superfields, these theories flow to either confining or Higgs vacua. Inherited duality for $\mathcal{N}=4$ super yang-mills (SYM) deformed by a single mass term has also been studied [24]. In this paper we conjecture that the $\mathcal{N}=1 \widehat{A}_{n-1}$ quiver gauge theories have $n S L(2, \mathbb{Z})$ duality symmetries distinct from Seiberg duality. These $S L(2, \mathbb{Z})$ groups do not commute and thus there is no sense in which we have a "diagonal" or "overall" $S L(2, \mathbb{Z})$. We establish that all these symmetries are inherited from their $\mathcal{N}=2$ parent.

\section{The $\mathcal{N}=2$ duality group}

In [4], Witten constructed four dimensional $\mathcal{N}=2, \widehat{A}_{n-1}$ quiver gauge theories by lifting a IIA brane construction to $M$-theory. The starting point is IIA with $n$ separated NS5-branes and $N$ D4-branes suspended between each of them. Specifically, the asymptotic coordinates of the $i$-th NS5-brane are $x^{7}=x^{8}=x^{9}=0$ and $x^{6}=x_{i}^{6}$. The $\mathrm{D} 4$-branes have four world volume coordinates in common with the NS5-branes $\left(x^{0}, \ldots, x^{3}\right)$, they also have finite extent in the $x^{6}$ direction thus the gauge theory living on their worldvolume is effectively four dimensional. There are $n$ gauge coupling constants, given by

$$
\frac{1}{g_{i}^{2}}=\frac{x_{i+1}^{6}-x_{i}^{6}}{8 \pi g_{s} L}
$$

The affine quiver gauge theories are engineered when the $x^{6}$ direction is periodic $^{1}$, with period, $L$. There are then $n$ NS- 5 branes, and the indices in (2.1) are taken cyclically, except that in going from $n$th brane to the first brane, one continues around the circle and picks up a full period so that

$$
\sum_{i=1}^{n} \frac{1}{g_{i}^{2}}=\frac{1}{8 \pi g_{s}} .
$$

Upon lifting to $M$-theory, the NS-5 branes will be separated in the $x^{10}$ direction as well and this separation is interpreted as the theta angle, namely

$$
\theta_{i}=\frac{x_{i+1}^{10}-x_{i}^{10}}{R} .
$$

The periodicity properties are now represented in terms of a torus, $E_{\tau}$, is constructed from the $x^{6}$ and $x^{10}$ directions. First, one must impose that $x^{10}$

\footnotetext{
${ }^{1}$ These are referred to as the elliptic models in [4]
} 
is periodic:

$$
x^{10} \sim x^{10}+2 \pi R,
$$

which guarantees the standard periodicity under $\theta_{j} \rightarrow \theta_{j}+2 \pi$ in each gauge theory factor separately. The periodicity in the $x^{6}$ direction is now modified to:

$$
\left(x^{6}, x^{10}\right) \sim\left(x^{6}+2 \pi L, x^{10}+\theta R\right)
$$

If one now defines:

$$
\begin{aligned}
p_{i} & \equiv \frac{i x_{i}^{6}}{16 \pi^{2} g_{s} L}+\frac{x_{i}^{10}}{2 \pi R}, \\
\tau & \equiv \frac{i}{8 \pi g_{s}}+\frac{\theta}{2 \pi},
\end{aligned}
$$

then the $p_{i}$ are points on the elliptic curve, $E_{\tau}$, with Teichmuller parameter, $\tau$. Individual gauge couplings are then differences between points on this torus, and the cyclically defined sum, (2.2), becomes

$$
\sum_{i=1}^{n}\left(p_{i+1}-p_{i}\right)=\tau .
$$

Specifically, because the D4-branes wrap the torus along the $x^{6}$-direction in the quiver gauge theory, we thus take:

$$
p_{n+1}=p_{1}+\tau, \quad p_{0}=p_{n}-\tau,
$$

We will discuss this below.

Witten thus concludes that the moduli space of coupling constants of the quiver gauge theory is the moduli space $\mathcal{M}_{1, n}$ of a 2 -torus with $n$ distinct, unordered punctures. The duality group of the four dimensional gauge theory is then the mapping class group $M(1, n)$.

\subsection{Generators and relations}

We now describe explicitly how this mapping class group acts on gauge couplings. The covering space of the moduli space $\mathcal{M}_{1, n}$ can then be parametrized by $\tau$ and the $n$ marked points $p_{i}$ in the complex plane. Since only differences of the $p_{i}$ have physical meaning, these $n$ points are only defined up to an overall shift:

$$
\left(\tau, p_{1}, \ldots, p_{n}\right) \sim\left(\tau, p_{1}+c, \ldots, p_{n}+c\right),
$$

for any complex constant, $c$. We shall call this basis for the representation of our group the point basis. Then the generators of $M(1, n)$ are the usual generators $S$ and $T$ of $M(1,1) \sim S L(2, \mathbb{Z})$, combined with permutations of 
the marked points, $s_{i}$ and individual lattice shifts $T_{i}$ and $t_{i}$. The explicit action of these generators are:

$$
\begin{aligned}
& S:\left(\tau, p_{1}, \ldots, p_{n}\right) \longrightarrow\left(-\frac{1}{\tau}, \frac{p_{1}}{\tau}, \ldots, \frac{p_{n}}{\tau}\right), \\
& T:\left(\tau, p_{1}, \ldots, p_{n}\right) \longrightarrow\left(\tau+1, p_{1}, \ldots, p_{n}\right), \\
& T_{i}:\left(\tau, p_{1}, \ldots, p_{n}\right) \longrightarrow\left(\tau, p_{1}, \ldots, p_{i}+1, \ldots, p_{n}\right), \\
& t_{i}:\left(\tau, p_{1}, \ldots, p_{n}\right) \longrightarrow\left(\tau, p_{1}, \ldots, p_{i}+\tau, \ldots, p_{n}\right), \\
& s_{i}:\left(\tau, p_{1}, \ldots, p_{i}, p_{i+1}, \ldots, p_{n}\right) \longrightarrow\left(\tau, p_{1}, \ldots, p_{i+1}, p_{i}, \ldots, p_{n}\right), \quad i \neq n, \\
& s_{n}:\left(\tau, p_{1}, \ldots, p_{n}\right) \longrightarrow\left(\tau, p_{n}-\tau, p_{2} \ldots, p_{n-1}, p_{1}+\tau\right) .
\end{aligned}
$$

The action of $s_{n}$ differs in form from the other $s_{i}$ because of the cyclic structure of the $p_{j}$ arising from the wrapping of the D4-branes. Indeed, one can obtain this formula if one properly applies the convention (2.9). This rule will also emerge from the discussion of gauge couplings, where we require that the permutations act in the same manner upon all gauge couplings.

There are non-trivial relations between these generators and some of the generators are actually redundant. First, note that $S$ and $T$ are the usual generators of $S L(2, \mathbb{Z})$ with $S^{2}$ acting non-trivially on the $n$-points but trivially on $\tau$. Less obvious are the relations obeyed by the other generators. To elucidate these we first introduce another basis for the representation called the gauge coupling basis. This basis is $\left(\tau_{1}, \ldots, \tau_{n}\right)$ where

$$
\begin{aligned}
\tau_{i} & =p_{i+1}-p_{i}, i \neq n, \\
\tau_{n} & =\tau+p_{1}-p_{n} .
\end{aligned}
$$

The overall gauge coupling constant $\tau$ can be recovered as $\tau=\sum_{i} \tau_{i}$. In this basis the modular group acts as follows ${ }^{2}$

$$
\begin{aligned}
S: & \left(\tau_{1}, \ldots, \tau_{n}\right) \longrightarrow\left(\frac{\tau_{1}}{\tau}, \ldots, \frac{\tau_{n-1}}{\tau}, \frac{\tau_{n}}{\tau}-1-\frac{1}{\tau}\right), \\
T: & \left(\tau_{1}, \ldots, \tau_{n}\right) \longrightarrow\left(\tau_{1}, \ldots, \tau_{n-1}, \tau_{n}+1\right), \\
T_{i} & :\left(\tau_{1}, \ldots, \tau_{n}\right) \longrightarrow\left(\tau_{1}, \ldots, \tau_{i-1}+1, \tau_{i}-1, \ldots, \tau_{n}\right), \\
t_{i}: & \left(\tau_{1}, \ldots, \tau_{n}\right) \longrightarrow\left(\tau_{1}, \ldots, \tau_{i-1}+\tau, \tau_{i}-\tau, \ldots, \tau_{n}\right), \\
s_{i}: & \left(\tau_{1}, \ldots, \tau_{i-1}, \tau_{i}, \tau_{i+1}, \ldots, \tau_{n}\right) \longrightarrow\left(\tau_{1}, \ldots, \tau_{i-1}+\tau_{i},\right. \\
& \left.\quad-\tau_{i}, \tau_{i+1}+\tau_{i}, \ldots, \tau_{n}\right)
\end{aligned}
$$

where we now interpret these formula with the exact cyclic convention: $\tau_{n+1}=\tau_{1}$ and $\tau_{0}=\tau_{n}$. This fixes the form of $s_{n}$ above.

\footnotetext{
${ }^{2}$ For $n=2$ the action of the $s_{i}$ is slightly different, as is familiar from the action of affine Weyl groups on fundamental weights.
} 
We can now find relations between these generators. Firstly, the $T_{i}$ and $t_{i}$ are related by the $S$-transformation

$$
t_{i}=S^{-1} T_{i} S \quad \text { and } \quad T_{i}^{-1}=S^{-1} t_{i} S .
$$

We introduce $\omega$, the generator of $\mathbb{Z}_{n}$, which in the gauge coupling basis acts as $\tau_{i} \rightarrow \tau_{i+1}, \forall i$ and can be easily seen to satisfy

$$
\omega=t_{i} s_{i-1} s_{i-2} \ldots s_{i+1} \text {. }
$$

We can build $T_{i}$ out of $T$ and the cyclic permutations $\omega$

$$
T_{i}=\omega^{n-i+1} T \omega^{-1} T^{-1} \omega^{-n+i} .
$$

Finally, the reflection operations $s_{i}$ are related by cyclic permutations

$$
s_{i+1}=\omega^{-1} s_{i} \omega \text {. }
$$

Thus we have shown that a minimal set of generators is $\left\{S, T, s_{1}, \omega\right\}$ although for a nice presentation we include $s_{i}, t_{i}, T_{i}$ and exclude $\omega$, see the appendix for such a presentation.

It is quite interesting that the $S L(2, \mathbb{Z})$ subgroup described above chooses a preferred node of the quiver. The $\mathbb{Z}_{n}$ subgroup rotates the nodes of the quiver and thus there are actually $n$ different $S L(2, \mathbb{Z})$ subgroups, all related by conjugation by an element of $\mathbb{Z}_{n}$. Note that the different $S L(2, \mathbb{Z})$ subgroups do not commute with each other. This means that there is no diagonal subgroup which could be the "overall" $S L(2, \mathbb{Z})$.

From the point of view of holographic RG flows in type IIB string theory this might seem a little strange at first. In type IIB theory there is only one $S L(2, \mathbb{Z})$ duality group in ten dimensions. However in a compactification of type IIB theory on an orbifold it is not clear how the $S L(2, \mathbb{Z})$ acts on the twisted sector fields. In order to determine that, one has to blow up the singularity, such that the whole geometry is smooth. Then the action of $S L(2, \mathbb{Z})$ is well defined. The stringy geometry provides $n$ different ways to blow up the singularity. These blowups are related by the $\mathbb{Z}_{n}$ quantum symmetry as we have discovered above. As expected, each $S L(2, \mathbb{Z})$ subgroup acts in the same way on the untwisted fields.

For a generic set of gauge couplings, it can happen that after one of the foregoing duality transformations one or more of the individual couplings have negative imaginary part. This is unphysical. So one must use these duality transformations with care. For example, after an $S$ transformation, the strength of the gauge couplings is determined by the differences of positions of NS5-branes in the $x^{6}$ direction. One can then use the involutions, $s_{i}$, to reorder the NS5-branes such that the $n$-tuple $\left(x_{1}^{6}, \ldots, x_{n}^{6}\right)$ is restored to increasing order. As we will see in the next section, the $s_{i}$ are, in fact, Seiberg dualities acting on the $i$-th node of the quiver theory. This is therefore in 
perfect accord with the previous observations [13] that Seiberg duality can be used to prevent $1 / g_{i}^{2}$ from becoming negative.

The mapping class group $M(1, n)$ has been studied in detail by Birman $[12,25]$. This group is an extension of the torus braid group $B_{n}\left(T^{2}\right)$ by $S L(2, \mathbb{Z})$. From the presentation of $B_{n}\left(T^{2}\right)$ given in [25] we can see that our representation is not faithful. If we supplement $B_{n}\left(T^{2}\right)$ with the relations $s_{i}^{2}=1$ we recover the presentation provided in the appendix. The non-trivial homeomorphism $s_{i}^{2}$ of $M(1, n)$ has become trivial, in the IIA picture this is due to brane-antibrane annihilation.

\section{The $\mathcal{N}=1$ duality group}

We now study deformations of the $\mathcal{N}=2$ quiver gauge theory by masses for the adjoint scalars. The methods of Leigh and Strassler [14] predict that, assuming the hypermultiplet masses are set to zero, the end-point of the resulting $\mathrm{RG}$ flow will be a non-trivial $\mathrm{CFT}[11,15]$.

\subsection{Small mass deformations}

To understand the $\mathcal{N}=1$ duality group we will again use the 5 -brane construction in $M$-theory. The construction of $\mathcal{N}=2$ gauge theories requires that the M5-brane wraps a complex curve that is embedded in the two complex dimensions with holomorphic coordinates $u=x^{4}+i x^{5}$ and $w=x^{6}+i x^{10}$. More generally, a $\mathcal{N}=1$ theory merely requires that the M5-brane wrap a curve embedded in three complex dimensions, with the extra dimension given by $v=x^{7}+i x^{8}$. In the IIA theory, the soft breaking of the $\mathcal{N}=2$ theory down to $\mathcal{N}=1$ supersymmetry can be achieved by essentially tilting the NS- 5 branes relative to one-another. In $M$-theory, we need the M5-brane to wrap a curve in $(u, v, w)$-space and for large $(u, v, w)$ the NS-5 branes must be asymptotic to points on the elliptic curve, $E_{\tau}$, defined by $(2.4),(2.5)$ in the $w$-direction and by straight lines in the $(u, v)$-direction. The directions of these lines can be parametrized by points, $z_{i}$, on a $\mathbb{P}^{1}$ that has $u$ and $v$ as homogeneous coordinates. Therefore, the asymptotic M5-brane data is the collections of points, $p_{i}$, on the torus and a set of points, $z_{i}$ on a $\mathbb{P}^{1}$.

As before, the gauge coupling constants $\tau_{i}$ are given by (2.11). When at least two of the NS5-branes, or equivalently the asymptotic legs of the M5-brane, fail to be parallel, $\left(z_{i} \neq z_{j}\right.$ for some $i$ and $\left.j\right)$ the $\mathcal{N}=2$ supersymmetry is softly broken by small masses $m_{i}$ for the adjoint scalars [18]. There is, however, a problem: given a quiver theory with $n$ nodes, there must be $n$ 
independent mass parameters for the $\mathcal{N}=1$ chiral multiplets in the $\mathcal{N}=2$ vector multiplets. However, a set of $n$ NS-5 branes has only $(n-1)$ independent relative angles between them, and so the tilting of branes describes all but one of the mass parameters. We will resolve this issue momentarily.

For a generic angle between the two NS5-branes, there is also an ambiguity in determining the adjoint scalar. If the branes are almost parallel, the canonical choice for the scalar is the position of the suspended D4-brane along the direction of the NS5-branes. However, there is a second, very heavy scalar for the motion of the suspended D4-branes perpendicular to the direction of the NS5-branes. For this reason, a mass formula valid for arbitrary masses should be a function on a double cover of the $\mathbb{P}^{1}$. The field theoretic meaning of the additional adjoint scalar which must be integrated in along the flow is unclear. At any rate, we will work primarily with the small mass formula. This means that all the branes are very nearly parallel to each other asymptotically.

The group acting in the $(u, v)$-directions that preserves the complex structure is $G L(2, \mathbb{C})$. In order to preserve the holomorphic 3 -form, this rotation group has to be restricted to $S L(2, \mathbb{C})$. Since the branes are all almost parallel, we can use the $S L(2, \mathbb{C})$ to rotate them so that they are all nearly asymptotic to the $v$-direction. This means that they can all be represented small values of the inhomogenous coordinates, $z_{i}=u_{i} / v_{i}$, on $\mathbb{P}^{1}$. The mass parameters, $m_{i}$, in the superpotential for the adjoint scalar $\Phi_{i}$ has to be a holomorphic function of $z_{i}$ and $z_{i+1}$. Further, the mass must vanish iff the two 5-branes are parallel. From this we get the following approximation when the masses are small:

$$
m_{i}=z_{i+1}-z_{i}
$$

Observe that the sum of the masses vanishes, and so, at least for small $m_{i}$, the missing mass parameter is the total, or global, mass parameter, $m=\sum_{i=1}^{n} m_{i}$.

In [4] a similar problem was encountered and solved for the independent hypermultiplet mass terms in the $\mathcal{N}=2$ theory. The solution was to fiber the mass parameters in a non-trivial manner over the torus: traveling around one the cycles of the torus resulted in a non-trivial (affine) translation of the masses. This affine shift, or connection on the fibration thus provided the non-trivial total mass. Here we use a similar solution, but we cannot use an affine fibration of the NS-5 branes as this would offset the D4-branes suspended between them, and thus generate unwanted hypermultiplet masses.

To generate a non-zero global mass we should fiber $\mathbb{C}^{2}$ described by $(u, v)$ over $E_{\tau}$, and only use the $S L(2, \mathbb{C})$ structure group, so as preserve the 
supersymmetry and fix the origin in $\mathbb{C}^{2}$ so as to avoid displacing the D4 branes. Since the global mass can be turned off continuously, the fibration has to be topologically trivial. The moduli space of such a fibration is classified by an embedding of $\pi_{1}\left(E_{\tau}\right)=\mathbb{Z} \oplus \mathbb{Z}$ into the maximal torus ${ }^{3}$ of $S L(2, \mathbb{C})$. If one considers the projective action of $S L(2, \mathbb{C})$ on the $\mathbb{P}^{1}$, then the obvious choice of maximal torus is to take the complexified rotations (rotations and scale transformations) $z \rightarrow a z$ that fix the origin and the point at infinity. However, this does not move branes located at the origin, and so we conjugate this torus so that the fixed points are $z=1$ and $z=-1$ on the $\mathbb{P}^{1}$. That is, we take the torus to be $z \rightarrow(a z+b) /(b z+a)$, with $a^{2}-b^{2}=1$. The effect of such transformations on points near $z=0$ is an affine shift: $z \rightarrow z+b / a$, and thus if one travels around the torus, one generates an overall shift in the global mass parameter.

We also see that for our purposes, we can approximate the $\mathbb{P}^{1}$ bundle by an affine fibration of $\mathbb{C}$ over $E_{\tau}$, which is incidentally also a principal $\mathbb{C}$ bundle. Our construction therefore closely parallels the construction of a hypermultiplet global mass via an affine fibration in [4].

A principal $\mathbb{C}$ bundle over $E_{\tau}=T^{2}$ has two gluing functions, one for each cycle of the base, which are encoded in

$$
\begin{aligned}
& \left(p_{i}, z_{i}\right) \sim\left(p_{i}+1, z_{i}+a\right), \\
& \left(p_{i}, z_{i}\right) \sim\left(p_{i}+\tau, z_{i}+b\right) .
\end{aligned}
$$

However, there is a gauge freedom that means that only one such parameter is physical. In general the gauge transformation means that we can shift the fiber coordinate by an arbitrary holomorphic function of the base variable: $z_{i} \rightarrow z_{i}^{\prime}=z_{i}+f\left(p_{i}\right)$. However in order to remain consistent with the constant gluing functions above, $f\left(p_{i}\right)$ must be a linear function. Further, the constant part of this linear function has no effect on the gluing functions and can be set to zero. Thus we have a gauge freedom: $g:(p, z) \rightarrow(p, z+c p)$ for some constant, $c$. This gauge transformation also modifies the gluing functions, and so we have:

$$
\begin{aligned}
\left(\tau, a, b, p_{i}, z_{i}\right) & \stackrel{g}{\longrightarrow}\left(\tau,-c \cdot 0+a+c \cdot 1,-c \cdot 0+b+c \cdot \tau, p_{i}, z_{i}+c p_{i}\right) \\
& =\left(\tau, a+c, b+c \tau, p_{i}, z_{i}+c p_{i}\right)
\end{aligned}
$$

By choosing $a=-c$ we get a trivial gluing function associated to the $\theta$-cycle of $T^{2}$, while the remaining gluing function is identified with the mass:

$$
\begin{aligned}
& m_{i}=z_{i+1}-z_{i}, \\
& m_{n}=z_{1}-z_{n}+m .
\end{aligned}
$$

\footnotetext{
${ }^{3}$ Note that the maximal torus of $S L(2, \mathbb{C})$ is $\mathbb{C}^{*}$.
} 
This periodicity now precisely parallels that of the gauge coupling constants. The gauge choice above (associating $m$ with the $x^{6}$ cycle) ensures, that $T_{i}$ acts as the identity on mass parameters as required by physics.

From the foregoing definition it is immediate that the masses transform in the same way as the gauge coupling constants under the permutation symmetries which generate the affine Weyl group (now with $\sum m_{i}=m$ as the level) when acting on the masses. In order to understand the action of $S$-duality on the mass parameters, we will need to explicitly work out gauge transformations in local coordinates.

The action of $S$-duality violates the gauge choice made above, and to restore the gauge it must be followed by a gauge transformation with $c=m$. The geometrical data thus transforms as:

$$
\begin{aligned}
\left(\tau, 0, m, p_{i}, z_{i}\right) & \stackrel{S}{\longrightarrow}\left(-\frac{1}{\tau}, m, 0, \frac{p_{i}}{\tau}, z_{i}\right) \\
\stackrel{g}{\longrightarrow} & \left(-\frac{1}{\tau}, 0, \frac{m}{\tau}, \frac{p_{i}}{\tau}, z_{i}-m \frac{p_{i}}{\tau}\right) .
\end{aligned}
$$

From now on we will leave implicit the fact that $S$-duality is coupled with the foregoing gauge transformation. It is instructive to check that $S^{2}$ acts in the following way

$$
\left(\tau, 0, m, p_{i}, z_{i}\right) \stackrel{S^{2}}{\longrightarrow}\left(\tau, 0,-m,-p_{i}, z_{i}\right)
$$

We now have the complete action of the duality group on the masses, namely

$$
\begin{aligned}
S: & \left(m, m_{1}, \ldots, m_{n}\right) \longrightarrow\left(\frac{m}{\tau}, m_{1}-\frac{m \tau_{1}}{\tau}, \ldots, m_{n-1}-\frac{m \tau_{n-1}}{\tau},\right. \\
& \left.m_{n}-\frac{m \tau_{n}}{\tau}+\frac{m}{\tau}\right), \\
t_{i}: & \left(m, m_{1}, \ldots, m_{i}, \ldots, m_{n}\right) \longrightarrow\left(m, m_{1}, \ldots, m_{i-1}+m, m_{i}-m, \ldots, m_{n}\right), \\
s_{i}: & \left(m, m_{1}, \ldots, m_{i-1}, m_{i}, m_{i+1}, \ldots, m_{n}\right) \\
& \quad \longrightarrow\left(m, m_{1}, \ldots, m_{i-1}+m_{i},-m_{i}, m_{i+1}+m_{i}, \ldots, m_{n}\right), \\
\omega: & \left(m, m_{1}, \ldots, m_{i}, \ldots, m_{n-1}, m_{n}\right) \longrightarrow\left(m, m_{2}, \ldots, m_{i+1}, \ldots, m_{n}, m_{1}\right)
\end{aligned}
$$

with the generators $\left\{T_{i}, T\right\}$ acting trivially.

We see from the foregoing construction that the $\mathcal{N}=1$ duality group is the same as the $\mathcal{N}=2$ duality group. This is partly due to the fact that the mapping class group of $\mathbb{P}^{1}$ is trivial, so no new symmetries are introduced in the $\mathcal{N}=1$ theory. There are still $n$ different $S L(2, \mathbb{Z})$ subgroups of the duality group, each related by conjugation by an element of $\mathbb{Z}_{n}$. 


\subsection{Seiberg duality}

For large mass deformations we must dispense with our geometric mass formula. However, at a scale below the smallest mass scale, we can integrate out all the massive fields and obtain a quartic superpotential for the hypermultiplet fields. In the IR we can expect a non-trivial CFT and here Seiberg duality acts non-trivially on couplings. As we will see, this action is in agreement with what we derived for the small mass approximation, namely they transform as Dynkin labels under an affine Weyl reflection and thus we interpret this duality "inherited" from its $\mathcal{N}=2$ parent theory.

It has long been appreciated that quartic superpotentials play a special role in Seiberg duality, namely the dual theory also has a quartic superpotential. This idea was used to great effect in the celebrated Klebanov and Strassler scenario [7], which is included in our analyis with $n=2$ and $m_{1}+m_{2}=0$. In the Klebanov-Strassler solution [7] the superpotential couplings are left invariant under Seiberg duality, this is a fixed point of a symmetry group elucidated in [15]. In general the superpotential couplings are not invariant under this operation.

We now review the action of Seiberg duality on the $\mathcal{N}=1$ superpotential. The $\mathcal{N}=2$ quiver has a unique renormalizable superpotential [8]:

$$
W_{\mathcal{N}=2}=\sum_{i=1}^{n} \lambda_{i} \Phi_{i}\left(A_{i} B_{i}-A_{i-1} B_{i-1}\right)
$$

where, for $\mathcal{N}=2$ supersymmetry $\lambda_{i}=\sqrt{2}$. We deform by the relevant operator

$$
\Delta W=\sum_{i=1}^{n} \frac{m_{i}}{2} \Phi_{i}^{2}
$$

which breaks supersymmetry to $\mathcal{N}=1$. Integrating out the massive adjoint fields results in a new superpotential

$$
W_{\mathcal{N}=1}=\sum_{i=1}^{n} h_{i}\left(A_{i} B_{i}-A_{i-1} B_{i-1}\right)^{2}
$$

where $h_{i}=-\lambda_{i}^{2} / m_{i}$.

There is a straightforward recipe for calculating the Seiberg dual theory [26]. When one gauge group becomes strongly coupled (relative to the groups of its adjacent nodes) one introduces dual quarks and "fundamental" mesons. 
To dualize on the $j$-th node one introduces a matrix of meson fields:

$$
M=\left(\begin{array}{cc}
A_{j-1} A_{j} & A_{j-1} B_{j-1} \\
B_{j} A_{j} & B_{j} B_{j-1}
\end{array}\right)
$$

and the superpotential (3.11) becomes

$$
\begin{aligned}
W_{\mathcal{N}=1}= & \sum_{i=1}^{j-2} h_{i}\left(A_{i} B_{i}-A_{i-1} B_{i-1}\right)^{2} \\
& +h_{j-1}\left(M_{12}^{2}+\left(B_{j-2} A_{j-2}\right)^{2}-2 M_{12} B_{j-2} A_{j-2}\right) \\
& +h_{j}\left(M_{12}^{2}+M_{21}^{2}-2 M_{11} M_{22}\right) \\
& +h_{j+1}\left(M_{21}^{2}+\left(A_{j+1} B_{j+1}\right)^{2}-2 M_{21} A_{j+1} B_{j+1}\right) \\
& +\sum_{i=j+2}^{n} h_{i}\left(A_{i} B_{i}-A_{i-1} B_{i-1}\right)^{2} \\
& +y\left(M_{11} a_{j-1} a_{j}+M_{12} a_{j-1} b_{j-1}+M_{21} b a_{j}+M_{22} b_{j} b_{j-1}\right),
\end{aligned}
$$

where we have also introduced "dual quarks", $\left(a_{j}, a_{j-1}, b_{j}, b_{j-1}\right)$. Since the action is quadratic in these new fields, one can trivially integrate them out. However, if the mesons, $M_{i j}$, are massive then they can be integrated out instead, and this results in a new quartic superpotential. This has the effect of making the following replacement of the fields attached to the $j$-th node:

$$
\left(A_{j}, A_{j-1}, B_{j}, B_{j-1}\right) \longrightarrow\left(a_{j}, a_{j-1}, b_{j}, b_{j-1}\right) .
$$

However, after integrating out the massive mesons the couplings undergo non-trivial transformations. If we abuse notation and rescale the "dual quarks" according to:

$$
\frac{y}{2 h_{j}}\left(a_{j}, a_{j-1}, b_{j}, b_{j-1}\right) \longrightarrow\left(A_{j}, A_{j-1}, B_{j}, B_{j-1}\right),
$$

then the resulting superpotential is

$$
W_{\mathcal{N}=1}=\sum_{i=1}^{n} h_{i}^{\prime}\left(A_{i} B_{i}-A_{i-1} B_{i-1}\right)^{2}
$$

where the couplings have transformed as follows,

$$
\begin{aligned}
{h_{i}^{\prime-1}}^{-1} & =h_{i}^{-1}, \quad i \neq j-1, j, j+1 \\
{h_{j-1}^{\prime}}^{-1} & =h_{j-1}^{-1}+h_{j}^{-1} \\
{h_{j}^{\prime-1}}^{-1} & =-h_{j}^{-1} \\
{h_{j+1}^{\prime}}^{-1} & =h_{j+1}^{-1}+h_{j}^{-1} .
\end{aligned}
$$


Recalling from [15] that the condition for a generalized conifold is $\sum h_{i}^{-1}=0$, one can see that the rescaling (3.15) is necessary for the Seiberg dual of a generalized confold to be a generalized conifold, for which there is much evidence [13]. We see from (3.17) that $h_{j}^{-1}$ transform under Seiberg duality as Dynkin labels of the $\widehat{A}_{n}$ Weyl group transform under a reflection.

This is in agreement with the transformations that we derived in the regime of small masses in the previous section. We know that the action of $T_{i}$ and $T$ is a symmetry of the $\mathcal{N}=1$ field theory, they act on gauge couplings in an identical way as for the $\mathcal{N}=2$ theory and they leave the superpotential couplings invariant. However it is desirable to provide a field theory derivation of the $S$-transformation. Once this is shown the action of $t_{i}$ is immediate. We leave this for future work.

\subsection{The cascade}

So far we have considered theories with product gauge groups with the rank of each group being equal. If one considers the $\mathcal{N}=1$ theories but with ranks that are no longer all equal, then a non-trivial RG flow ensues [16]. This flow can be adequately described by a cascade of Seiberg dualities on alternate nodes. In [13] it was realized that the effect of this RG flow on gauge couplings can be simply described by the motion of a "billiard ball" in the fundamental Weyl chamber of the $A_{n-1}$ affine Weyl group. When the ball bounces off a wall this corresponds to a Weyl reflection or Seiberg duality. From the results of [15] we see that in addition, Seiberg duality acts on superpotential couplings as a fundamental affine Weyl reflection. One main result from [15] was that there exists universality in the IR of a general cascade associated to the $\widehat{A}_{n}$ quiver. More precisely, define a UV theory to be one of the $\mathcal{N}=1$ theories discussed above but with the ranks of the gauge groups unequal and an arbitrary initial set of superpotential couplings $h_{i}$. Define

$$
h^{-1} \equiv \sum_{i=1}^{n} h_{i}^{-1},
$$

and observe that this quantity is invariant under the actions of Seiberg dualities (3.17). Consider the projective space with homogeneous coordinates $\left[h_{1}^{-1}, \ldots, h_{n}^{-1}\right]$, then a sequence of Seiberg dualities taken around the quiver has the effect of translation along this projective space. There is a submanifold of the projective space defined by $h^{-1}=0$ and called the "conifold subspace". Apart from being invariant under Seiberg duality, this subspace is an attractor for all duality cascades [15]. Consider a theory with $h^{-1} \neq 0$, 
then after a large number of such Seiberg dualities a subset of superpotential couplings will satisfy $\left|h_{i}^{-1}\right| \gg h^{-1}$. As explained in [15], the physical couplings are the projective quantities, $h_{i} / h_{j}$, and since $h^{-1}$ is invariant, the physical couplings are actually approaching the conifold subspace.

This property has a simple explanation in terms of paths in the affine Weyl group. The couplings $h_{i}^{-1}$ are Dynkin labels for the reflection group, and $h^{-1}=\sum_{i=1}^{n} h_{i}^{-1}$ is the level. Consider the $\widehat{A}_{1}$ Weyl group with Dynkin labels $\lambda_{1}, \lambda_{2}$ and level $\lambda$. Under a Weyl reflection the level is left invariant, and furthermore the pure translations in the affine Weyl group are proportional to the level. If we draw the line $\lambda_{1}+\lambda_{2}=\lambda$ in the plane as in Figure 1, then a Weyl reflection is a reflection in a hyperplane which passes through either of the two points $(0, \lambda)$ or $(\lambda, 0)$ and is perpendicular to this line. From the figure we see that alternating reflections result in an arbitrary initial point (here we chose an initial point to lie between $(\lambda, 0)$ and $(0, \lambda))$ being mapped toward infinity. In projective space this point is moving toward the point $\lambda_{1} / \lambda_{2}=-1$. As explained in [15] it is the projective coordinates

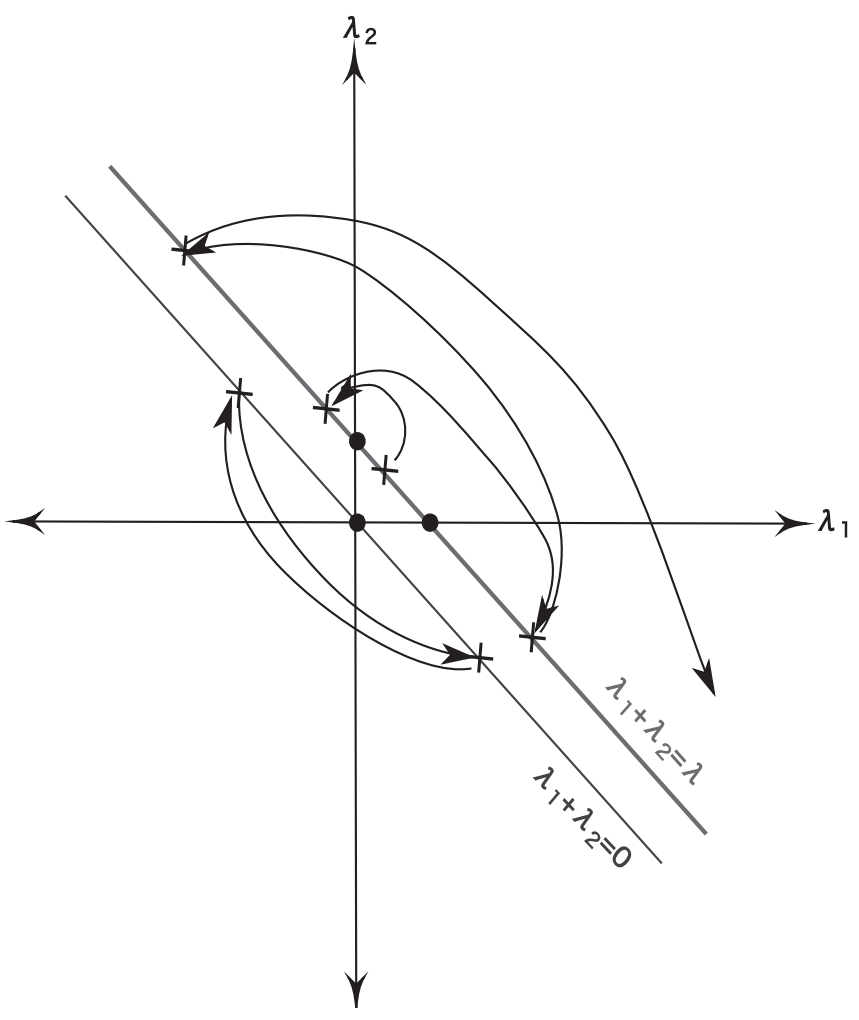

Figure 1: Weyl reflections acting on fundamental weights of $\widehat{A}_{1}$. 
$\left[h_{1}^{-1}, h_{2}^{-1}\right]=\left[\lambda_{1}, \lambda_{2}\right]$ that are important parameters. If the initial point was chosen to lie on the line with $\lambda=0$ then the two reflection planes coincide: the translations collapse because the level is zero. This is the well known fact that only the subgroup of the affine Weyl group which is isomorphic to the finite Weyl group acts faithfully on roots, and for $\widehat{A}_{1}$ this means that the point remains at $\lambda_{1} / \lambda_{2}=-1$. This is a simple explanation for the universality found in [15].

\section{Discussion}

One interesting open problem that we have not addressed in this paper is the relationship between the duality group found in the M-theory construction and the $S U(1, n)$ found in five dimensional gauged supergravity. The M-theory duality group is the symmetry group of a quiver gauge theory with finite rank gauge group and we expect that it admits an embedding into $S U(1, n)$ in the same sense that $S L(2, \mathbb{Z})$ embeds into $S U(1,1)$. There are certainly $n$ distinct, natural, non-commuting $S L(2, \mathbb{Z})$ subgroups in $S U(1, n)$, and it is tempting to identify them with the subgroups identified above. One can also embed the $A_{n-1}$ affine Weyl group into $S U(1, n)$. This is straightforward if one adds another dimension to the weight spaces. This extra dimension is the $L_{0}$ operator familiar from WZW models, but it is not clear how this relates to the gauged supergravity. Indeed, while parts of the duality group identified here do fit into the $S U(1, n)$ of supergravity, we have not yet succeeded in embedding the entire duality group in a manner consistent with signatures of the appropriate invariant metrics. In fact, this may not be possible, since it is perhaps naive to hope that supergravity captures all the dualities of the field theory.

There is a fascinating connection to WZW models which may not be unrelated. The affine Weyl group and $\mathbb{Z}_{n}$ appear naturally in Kac-Moody algebras but further, the parameters in the characters have modular transformations identical to those found here for gauge couplings. Obviously a fundamental input to defining a character is to have a well defined $L_{0}$ operator, something we are lacking here. Moreover, the familiar unitary representations of WZW models require an integer level, whereas the level, $\tau$, for the field theory is an arbitrary complex number.

There is also the connection between WZW models, the mapping class group of Riemann surfaces with punctures and Chern-Simons theory [27, 28 . It would be fascinating to find a connection between the F-terms of the four-dimensional quiver gauge theory and Chern-Simons theory in three dimensions, perhaps along the lines [29-31]. 


\section{Acknowledgments}

This work is supported in part by funds provided by the DOE under grant number DE-FG03-84ER-40168. The work of N.H. is supported in part by a Fletcher Jones Graduate Fellowship from USC. We would like to thank David Berenstein, Richard Corrado, Ruth Corran, Mike Douglas, Chris Herzog, Timothy Hollowood, Ken Intrilligator, Fyodor Malikov, Bob Penner, Johannes Walcher and Ed Witten for useful discussions and correspondence.

\section{A Presentation of the $\mathcal{N}=2$ duality group}

Here we write down a presentation for the $\mathcal{N}=2$ duality group. The generators $\left\{s_{i}, t_{j}, T_{j} \mid i=1, \ldots, n-1 j=1, \ldots, n\right\}$ are also generators of $B_{n}\left(T^{2}\right)$, the torus braid group on $n$ strands. When we include the relations $s_{i}^{2}=1$, the more complicated relations in $B_{n}\left(T^{2}\right)$ collapse (see [25] for a presentation of $B_{n}\left(T^{2}\right)$ ) leaving only the relations we have supplied here.

Firstly, we have $S L(2, \mathbb{Z})$ generated as usual by $\{S, T\}$, note that $S^{2}$ is non-trivial

$$
S^{4}=1, \quad S^{2} T=T S^{2}, \quad(S T)^{3}=1 .
$$

The braid group has only $n-1$ permutations but after imposing $s_{i}^{2}=1$ we can build one more permutation which enhances the symmetric group into the $\widehat{A}_{n-1}$ affine Weyl group,

$$
\begin{aligned}
s_{n} & =t_{1}^{-1} t_{n} s_{1} s_{2} \ldots s_{n-1}, \\
s_{i}^{2} & =1 \\
s_{i} s_{i+1} s_{i} & =s_{i+1} s_{i} s_{i+1} \forall i(n \neq 2) \\
s_{i} s_{j} & =s_{j} s_{i}, \quad|i-j|>1 .
\end{aligned}
$$

Taken together (A.4) and (A.5) give the canonical presentation of $\widehat{A}_{n-1}$. Other relations within the braid generators are

$$
\begin{aligned}
t_{i} T_{j} & =T_{j} t_{i}, \\
s_{i} t_{i} s_{i} & =t_{i+1}, \quad s_{i} t_{i+1} s_{i}=t_{i}, \\
s_{i} T_{i} s_{i} & =T_{i+1}, \quad s_{i} T_{i+1} s_{i}=T_{i} \forall i .
\end{aligned}
$$


As mentioned above, the mapping class group is an extension of $B_{n}\left(T^{2}\right)$ by $S L(2, \mathbb{Z})$, explicitly this is given by

$$
\begin{aligned}
& S^{-1} s_{i} S=s_{i}, \quad i \neq n, \\
& T^{-1} s_{i} T=s_{i}, \quad i \neq n, \\
& S^{-1} T_{i} S=t_{i}, \quad S^{-1} t_{i} S=T_{i}^{-1}, \quad \forall i, \\
& T^{-1} t_{i} T=T_{i} t_{i}, \quad T^{-1} T_{i} T=T_{i} \forall i .
\end{aligned}
$$

\section{References}

[1] J.M. Maldacena, The large $N$ limit of superconformal field theories and supergravity, Adv. Theor. Math. Phys. 2 (231) (1998) [Int. J. Theor. Phys. 38 (1113) (1999)]; arXiv:hep-th/9711200.

[2] A. Klemm, W. Lerche, P. Mayr, C. Vafa and N.P. Warner, Self-dual strings and $N=2$ supersymmetric field theory, Nucl. Phys. B 477 (746) (1996); arXiv:hep-th/9604034.

[3] A. Hanany and E. Witten, Type IIB superstrings, BPS monopoles, and three-dimensional gauge dynamics, Nucl. Phys. B 492 (152) (1997); arXiv:hep-th/9611230.

[4] E. Witten, Solutions of four-dimensional field theories via $M$-theory, Nucl. Phys. B 500 (3) (1997); arXiv:hep-th/9703166.

[5] D.Z. Freedman, S.S. Gubser, K. Pilch and N.P. Warner, Renormalization group flows from holography supersymmetry and a c-theorem, Adv. Theor. Math. Phys. 3 (363) (1999); arXiv:hep-th/9904017.

[6] K. Pilch and N.P. Warner, $\mathrm{N}=1$ supersymmetric renormalization group flows from IIB supergravity, Adv. Theor. Math. Phys. 4 (627) (2002); arXiv:hep-th/0006066.

[7] I.R. Klebanov and M.J. Strassler, Supergravity and a confining gauge theory: duality cascades and chiSB-resolution of naked singularities, JHEP 0008 (052) (2000); arXiv:hep-th/0007191.

[8] M.R. Douglas and G.W. Moore, D-branes, quivers, and ALE instantons, arXiv:hep-th/9603167.

[9] S. Kachru and E. Silverstein, 4d conformal theories and strings on orbifolds, Phys. Rev. Lett. 80 (4855) (1998); arXiv:hep-th/9802183.

[10] G. Dall'Agata, C. Herrmann and M. Zagermann, General matter coupled $\mathrm{N}=4$ gauged supergravity in five dimensions, Nucl. Phys. B 612 (123) (2001); arXiv:hep-th/0103106. 
[11] R. Corrado, M. Gunaydin, N.P. Warner and M. Zagermann, Orbifolds and flows from gauged supergravity, Phys. Rev. D 65 (125024) (2002); arXiv:hep-th/0203057.

[12] J.S. Birman, Braids, links, and mapping class groups, Ann. Math. Stud., (82), Princeton University Press, 228 p.

[13] F. Cachazo, B. Fiol, K.A. Intriligator, S. Katz and C. Vafa, A geometric unification of dualities, Nucl. Phys. B 628 (3) (2002); arXiv:hepth/0110028.

[14] R.G. Leigh and M.J. Strassler, Exactly marginal operators and duality in four-dimensional $\mathrm{N}=1$ supersymmetric gauge theory, Nucl. Phys. B 447 (95) (1995); arXiv:hep-th/9503121.

[15] R. Corrado and N. Halmagyi, $N=1$ field theories and fluxes in IIB string theory, arXiv:hep-th/0401141.

[16] I.R. Klebanov and E. Witten, Superconformal field theory on threebranes at a Calabi-Yau singularity, Nucl. Phys. B 536 (199) (1998); arXiv:hep-th/9807080.

[17] S. Gubser, N. Nekrasov and S. Shatashvili, Generalized conifolds and four dimensional $\mathrm{N}=1$ superconformal theories, JHEP 9905 (003) (1999); arXiv:hep-th/9811230.

[18] J.L.F. Barbon, Rotated branes and $\mathrm{N}=1$ duality, Phys. Lett. B 402 (59) (1997); arXiv:hep-th/9703051.

[19] S. Elitzur, A. Giveon and D. Kutasov, Branes and $\mathrm{N}=1$ duality in string theory, Phys. Lett. B 400 (269) (1997); arXiv:hep-th/9702014.

[20] See Matt Strassler's 2003 TASI lectures for a nice exposition.

[21] P.C. Argyres, M.R. Plesser and N. Seiberg, The moduli space of N = 2 SUSY QCD and duality in N = 1 SUSY QCD, Nucl. Phys. B 471 (159) (1996); arXiv:hep-th/9603042.

[22] N. Dorey and S.P. Kumar, Softly-broken N = 4 supersymmetry in the large-N limit, JHEP 0002 (006) (2000), arXiv:hep-th/0001103.

[23] N. Dorey, T.J. Hollowood and S. Prem Kumar, An exact elliptic superpotential for $\mathrm{N}=1^{*}$ deformations of finite $\mathrm{N}=2$ gauge theories, Nucl. Phys. B 624 (95) (2002); arXiv:hep-th/0108221.

[24] P.C. Argyres, K.A. Intriligator, R.G. Leigh and M.J. Strassler, On inherited duality in $\mathrm{N}=1 \mathrm{~d}=4$ supersymmetric gauge theories, JHEP 0004 (029) (2000); arXiv:hep-th/9910250.

[25] J.S. Birman, On braid groups, Comm. Pure Appl. Math. (22) (1968) $41-72$.

[26] N. Seiberg, Electric - magnetic duality in supersymmetric nonabelian gauge theories, Nucl. Phys. B 435 (129) (1995); arXiv:hep-th/9411149. 
[27] G.W. Moore and N. Seiberg, Polynomial equations for rational conformal field theories, Phys. Lett. B 212 (451) (1988).

[28] G.W. Moore and N. Seiberg, Lectures On Rcft, Published in Trieste Super-strings, 1989: 1-129.

[29] E. Witten, Chern-Simons gauge theory as a string theory, Prog. Math. 133 (637) (1995); arXiv:hep-th/9207094.

[30] M. Bershadsky, S. Cecotti, H. Ooguri and C. Vafa, Kodaira-Spencer theory of gravity and exact results for quantum string amplitudes, Commun. Math. Phys. 165 (311) (1994); arXiv:hep-th/9309140.

[31] R. Dijkgraaf and C. Vafa, A perturbative window into non-perturbative physics, arXiv:hep-th/0208048. 
